\title{
La transversalidad en la enseñanza de la medicina: el ejemplo de la Oncología
}

\author{
A. Barnadas-Molins ${ }^{a, b}$, J.E. Baños ${ }^{c, d}$
}

Introducción. El desarrollo de la medicina durante el siglo xIx conllevó la aparición de las especialidades médicas y quirúrgicas, configurando un nuevo escenario asistencial con la fragmentación de los tradicionales servicios de medicina y cirugía. Estos cambios también acontecieron en el campo de la docencia de la medicina, originando algunos conflictos en la definición de competencias que aún persisten. La patología médica debería actuar como punto de encuentro y eje vertebrador del resto de las especialidades médicas, pero hasta hace relativamente poco tiempo ha existido un excesivo esfuerzo en delimitar el campo de cada disciplina, aspecto que no ha facilitado una comunicación fluida entre los diferentes especialistas. La atención a los pacientes con cáncer necesita de la implicación y colaboración de los diferentes profesionales que participan en el diagnóstico y tratamiento y, por ello, precisa de un diálogo fluido entre todos ellos. La oportunidad de poder desarrollar en el pregrado una formación multidisciplinar que englobe aspectos referentes a la integración de los diferentes especialistas en beneficio del paciente facilitará una mejora en la optimización de los recursos sanitarios. La enseñanza de la Oncología en el pregrado ha estado compartida con otras especialidades, pero hasta el momento actual no ha tenido la adecuada consideración. La Oncología constituye un claro ejemplo de que el trabajo en equipo permite mejorar la eficiencia y conducción de los procesos asistenciales. Es de esperar que en las futuras promociones de estudiantes existan menos resistencias para establecer puntos de encuentro y de sinergia y que ello redunde en la adquisición de una visión más amplia y posibilista, y que pueda aplicarse en beneficio de los futuros enfermos.

Palabras clave. Docencia de la Oncología. Espacio Europeo de Educación Superior. Enseñanza de la medicina. Oncología médica.

\section{Cross-sectional approach in medical education: the example of Oncology}

The development of Medicine throughout the 19th century led to the appearance of medical and surgical specialties, which thus gave rise to a new health care scenario in which the traditional services of medicine and surgery became fragmented. These changes also came about within the field of the teaching of medicine and gave rise to a number of conflicts over the definition of competencies that still exist today. Medical pathology should act as a point of contact and backbone for the other medical specialities, but up until a relatively short time ago too much effort was being made to define the limits of each discipline, something that has not encouraged very fluent communication among the different specialists. Health care for cancer patients requires the commitment and collaboration of the different professionals who are involved in the diagnosis and treatment of the disease, and this in turn calls for fluent dialogue among all of them. The chance to be able to carry out multidisciplinary training for under-graduates that covers aspects related with the integration of the different specialists in order to benefit patients will make it easier to optimise health care resources. The teaching of Oncology at undergraduate level has been shared with other specialties, but up until now it has been paid too little attention. Oncology is a clear example of how teamwork makes it possible to improve the efficiency of health care processes and the way they are implemented. It is to be expected that in the future students will meet less resistance to establishing points of contact and synergy, and that this will result in their acquiring a broader and more positive vision, which they will be able to apply to help their patients.

Key words. European Higher Education Area. Medical oncology. Teaching medicine. Teaching oncology. a Servicio de Oncología Médica. Hospital de la Santa Creu i Sant Pau.

b Departamento de Medicina. Universidad Autónoma de Barcelona. Laboratorio de Neurofarmacología. Departamento de Ciencias Experimentales y de la Salud. Universitat Pompeu Fabra.

d Parc de Recerca Biomèdica de Barcelona. Barcelona, España.

\section{Correspondencia}

Dr. Agustí Barnadas. Servicio de Oncología Médica. Hospital de la Santa Creu i Sant Pau. Sant Antoni M. Claret, 167. E-08025 Barcelona.

Fax +34 935565679 .

E-mail abarnadasm@santpau.cat 


\section{Introducción}

El ingente aumento del conocimiento médico derivado del desarrollo de la medicina científica durante el siglo xIX conllevó la aparición de las especialidades médicas y quirúrgicas para permitir la aplicación de los nuevos paradigmas y las innovaciones tecnológicas [1]. Su desarrollo acabó delimitando un nuevo escenario asistencial a lo largo del siglo xx en el que los tradicionales servicios de medicina y cirugía fueron fragmentándose cada vez más [2,3]. Esta situación se trasladó progresivamente a la docencia médica con los inevitables conflictos de competencias y sigue sin resolverse en la actualidad, como muestran algunas de las tensiones en la redacción de los nuevos planes de estudio que deben adaptarse al Espacio Europeo de Educación Superior (EEES) [4-8]. En el presente artículo se reflexionará sobre la importancia docente de la patología médica como elemento integrador de las especialidades médicas, así como de la necesidad de aportar un nuevo modelo que permita la presentación más eficaz de los problemas de salud, en este caso aplicado al caso de la docencia de la Oncología.

\section{Algunas limitaciones de los planes de estudio actuales}

La educación médica en España ha utilizado durante décadas un modelo en el que los contenidos de las diferentes disciplinas se articulaban en materias que se presentaban de forma independiente $y$, con frecuencia, más como una yuxtaposición que como una secuencia razonable de materias o asignaturas. Dicho de otra forma, existía una especie de 'compartimentación' del conocimiento que se avenía poco con la realidad de la práctica médica. Se dejaba para fases posteriores de la educación, ya fuera en el periodo de rotatorio del último año o la propia formación posgraduada, la posibilidad de que el estudiante realizara un proceso de síntesis de toda la formación recibida para poder aplicarla a su actividad diaria. Este modelo docente tenía numerosos problemas como, por ejemplo, la separación innecesaria de disciplinas básicas y clínicas (con la percepción del estudiante de que las primeras eran un mal necesario para llegar a las segundas), la repetición a veces contradictoria de contenidos (la hipertensión explicada en patología general, cardiología y nefrología) o secuencias inadecuadas de éstos (explicación de los antiepilépticos antes de la epilepsia). Además, algunas asignaturas se encontraban desubicadas, como la bioestadística en primer curso, cuando los estudiantes eran incapaces de entender su utilidad en medicina. En otras ocasiones, la radiología de tercer curso explicaba la semiología radiológica del enfisema o la neumonía, cuando los estudiantes no habían recibido la formación básica sobre estas entidades clínicas.

El deseo de corregir estas limitaciones ha llevado al empleo de diversas estrategias docentes como la organización curricular basada en la integración de las disciplinas o el aprendizaje basado en problemas (ABP) [9]. Utilizados ampliamente en las universidades anglosajonas y escandinavas, su implantación en España ha sido mucho menor. Ello no significa que en España se intentaran modelos educativos innovadores, como el de la Universidad de Alacant, que se abandonaron progresivamente [10]. Un ejemplo vivido por los autores fue el de la Universitat $\mathrm{Au}$ tònoma de Barcelona (UAB) donde, a mediados de la década de los noventa, estuvo en vigor un plan de estudios con una integración parcial de disciplinas. A pesar de que los resultados académicos fueron buenos, se sustituyó poco después por otro de características tradicionales [11]. El ABP sólo se ha implantado de forma generalizada en la Facultad de Medicina de la Universidad de Castilla La Mancha [12], aunque han existido experiencias parciales en otras (UAB $[13,14]$, Zaragoza [15]). Las razones que podrían explicar la falta de éxito de las innovaciones educativas en el ámbito médico son múltiples, aunque es posible que uno de los motivos más importantes sea la existencia de una cultura académica propia en las facultades de Medicina españolas. Ésta reforzaría la identidad disciplinar y rechazaría los modelos de integración en que los profesores tienen la percepción de que carecen del control sobre la asignatura que imparten. Los modelos de integración curricular despertarían la sospecha de que podrían obviar aspectos relevantes y propios de cada disciplina que los docentes creen indispensables para la formación médica. En consecuencia, se prefiere el modelo donde la transversalidad está prácticamente ausente de los planes de estudio, lo que ignora la situación 
real de la práctica asistencial, cuando se precisa la integración para realizar una actividad clínica adecuada. En realidad, la evolución de la medicina hacia la especialización, apuntada al principio de este artículo, no ha hecho más que agravar la situación al proceder a una 'parcelización' de la actividad clínica que se ha correspondido en el ámbito docente con una pérdida de la visión holística de la actividad médica.

\section{La patología médica como oportunidad}

La patología médica, como traslación académica de la medicina interna, es una disciplina fundamental en la enseñanza de la Medicina y ejemplifica los problemas señalados en las secciones anteriores. En los últimos cincuenta años, la progresiva complejidad de la medicina clínica y de la atención hospitalaria ha conducido a una progresiva subespecialización. Este proceso ha generado el temor de que las funciones tradicionales de la medicina interna se vaciaran de contenido y que fueran sus especialidades las que la asumieran en su práctica totalidad. Los grandes internistas de las décadas pasadas han sido desplazados por aquellos que se han dedicado a una parte de la medicina interna original para desarrollar mejor su asistencia, docencia e investigación. El proceso ya había empezado cuando Osler publicó su Principles and practice of medicine [16] y se acentuó de forma imparable a lo largo del siglo $\mathrm{xx}$.

A finales de la década de los sesenta, Farreras apuntaba en la séptima edición de su conocida Medicina interna [17]: 'Hoy se saben demasiados datos, hechos y matices clínicos para que quepan en orden y sin confusiones en un solo cerebro. Disertar con autoridad sobre todos ellos es una utopía. La cuestión de cómo tratar esta situación de forma eficiente fue abordada pocos años después en la novena edición por el sucesor de Farreras, Ciril Rozman, quien señalaba las peligrosas tensiones que podían aparecer entre los que llamaba 'internistas generales' e 'internistas especialistas' [18]. Para Rozman, 'en este amplio, complejo y bellísimo campo de la medicina que es la medicina interna, tan sólo los esfuerzos aunados de un conjunto de hombres con dedicación forzosamente diversificada permitirá realizar la triple función: asistencial, docente e investigadora, que con pleno derecho reclama la sociedad para sí, sea de la máxima calidad' [18]. A pesar de los años transcurridos, el debate sigue aún abierto, como demuestra el reciente artículo de Foz [19], un análisis detallado del problema con una buena perspectiva histórica.

La preocupación descrita para la medicina interna puede aplicarse también a la patología médica. ¿Debe ésta enseñarse por internistas o reflejar la evolución natural de la disciplina? De nuevo las palabras de Rozman tienen su aplicación, pues es difícil pensar en la docencia de la disciplina por los diferentes especialistas médicos sin la presencia de los internistas generales. Sin embargo, no es menos cierto que la complejidad de la medicina moderna sugiere que los primeros puedan y deban participar en la docencia de la patología médica para permitir que los estudiantes se formen de forma óptima en sus diferentes áreas, especialmente en aquellas que, por su especialización, no suelen ser ocupación prioritaria de los internistas.

El borrador de la Orden Ministerial que regula los estudios de medicina adaptados al EEES recomendaba explícitamente la organización de la docencia de forma integradora y establecía que 'el plan de estudios, considerado el carácter transversal de las competencias, reflejará que los contenidos se integren horizontal y verticalmente, facilitando el contacto precoz con las disciplinas clínicas' [20]. Curiosamente, este redactado desapareció totalmente de la Orden Ministerial definitiva [21], aunque las competencias seguían siendo básicamente las mismas. Terés et al [22] también han defendido la existencia de una troncalidad en los estudios que permita que los estudiantes obtengan una formación amplia y común en el ámbito de aquellas competencias que comprenderían este currículo nuclear. Resulta evidente que, frente a la subespecialización excesiva, es preciso dotar a los estudiantes de una formación básica y central. Ello no es óbice para que los médicos especialistas puedan tener un lugar en la enseñanza de esa formación troncal, ni para que puedan disponer de un espacio docente para presentar los principios básicos de su especialidad, sobre todo en aquellas disciplinas que tratan enfermedades de elevada prevalencia que cualquier médico debería conocer con cierto detalle.

La docencia de la patología médica debería evitar las controversias que suelen ser comunes en la práctica asistencial. Por ejemplo, no es 
aconsejable que el debate de si la hipertensión es atribución de internistas, cardiólogos o nefrólogos llegue a la docencia. En la nueva situación donde el tiempo dedicado a la formación teórica se reducirá claramente, parece obvio que no se repita la explicación de una enfermedad en varias ocasiones de forma no siempre coincidente. De forma similar, las enfermedades de afectación multisistémica, como las colagenosis, deberían enseñarse de forma integrada y no de forma parcial según el órgano afectado. En el caso del cáncer de pulmón, ¿quién tiene la atribución docente, los neumólogos, los oncólogos, los internistas o los cirujanos torácicos? Estos dos ejemplos pretenden ilustrar sobre un problema que es generalizable a gran parte de la patología médica, pero también a todo el plan de estudios del nuevo Grado de Medicina.

En el ámbito docente, la patología médica debería actuar como la disciplina integradora que fue en el pasado. En la actualidad, la tradicional tentación centrífuga de la docencia debería evitarse y la disgregación que supondría una ausencia de coordinación entre los diferentes ámbitos de la disciplina sólo puede conducir a perjuicios para los estudiantes. Esta situación se hará especialmente patente cuando los profesores deban reducir la carga teórica de sus disciplinas a causa de la adaptación al EEES. Tal proceso no debe considerarse como un obstáculo, sino más bien como una gran oportunidad de revisar los programas de las diferentes asignaturas, proceder a la supresión de lo reiterativo, la integración de lo que sea posible, la secuenciación racional y la relación armónica entre materias básicas y clínicas. La nueva estructura de los planes de estudio basada en competencias que no se atribuyen a las clásicas áreas de conocimiento debería facilitar esta posibilidad. Sería utópico, sin embargo, asumir que estas recomendaciones serán de fácil aceptación dadas las tensiones existentes en las facultades de Medicina, como han señalado recientemente Torres et al [23].

\section{El ejemplo de una integración: la Oncología como disciplina longitudinal y transversal}

Es de sobras conocido que el cáncer constituye un problema de salud de gran magnitud, pero la preparación de los futuros médicos para afrontar este tipo de enfermedades es generalmente insuficiente. Esta limitación adquiere una especial relevancia en aquellos profesionales que deban ejercer en un ámbito de asistencia primaria y rural, con mayor dificultad para acceder a los recursos de instituciones con un elevado nivel de complejidad [24,25]. Por otra parte, en estas circunstancias, los futuros profesionales de la salud no están suficientemente capacitados para asumir las demandas y expectativas de la sociedad en la que se encuentran y que a su vez ha contribuido a que su formación fuera una realidad.

Hasta el momento actual, la enseñanza de las enfermedades cancerosas se ha efectuado en el seno de diferentes asignaturas y de forma escasamente coordinada, parcelada, repetitiva, en ocasiones contradictoria y, pese a todo, insuficiente. En España es reveladora la frase de Eduardo Díaz-Rubio: 'esta enseñanza no coordinada conduce al pesimismo frente al cáncer, no sólo improcedente sino altamente peligroso, saliendo en definitiva el estudiante de la universidad con la idea de la incurabilidad del cáncer. Es realmente inconcebible que la mayoría de los médicos de nuestro país tengan una idea tan negativa respecto a las posibilidades terapéuticas del cáncer, que a veces en nada se diferencian de la que tiene el hombre de la calle' [26].

Por ello, en las enfermedades oncológicas, esta necesidad de integración docente es básica y refleja la adaptación de la medicina a los nuevos tiempos. Hace algunas décadas, los tumores malignos se consideraban una afectación exclusivamente local que debía eliminarse por medios quirúrgicos o radioterapia. Sin embargo, pronto se vio que el problema real de la enfermedad no era tanto la afectación local sino la posibilidad de diseminación sistémica por las metástasis que, para la mayoría de los pacientes, supone la amenaza vital más importante. Con ello, en muchos casos, el cáncer dejó de ser una enfermedad local para convertirse en una enfermedad sistémica. Durante décadas, la falta de medicamentos eficaces siguió dejando el tratamiento en manos de los cirujanos y de los especialistas en radioterapia. Sin embargo, a partir de la década de los cincuenta, la aparición de medicamentos eficaces permitió actuar farmacológicamente. Más tarde, los conocimientos sobre citocinética tumoral y la biología molecular del tumor supusieron un grado de complejidad asistencial que pronto precisó 
de médicos que se dedicaran de forma exclusiva a estas enfermedades en estrecha colaboración con otros especialistas. El cáncer de mama es un buen ejemplo de la nueva situación, en la que diversos especialistas participan en las diversas fases de la enfermedad de forma coordinada y eficaz. Por sus características clínicas, son los médicos generales o los ginecólogos quienes realizan la primera sospecha diagnóstica. Apoyados por los especialistas en el diagnóstico por la imagen y por los patólogos, se confirma el diagnóstico. Tras una valoración conjunta en el seno de un comité específico o mediante el seguimiento de una guía clínica se establece la propuesta terapéutica más adecuada. En la mayor parte de los casos son los ginecólogos o los cirujanos quienes efectúan el primer tratamiento extirpando el tumor y el estudio de los ganglios de drenaje del mismo. Tras su recuperación, la paciente pasa a la consulta de Oncología donde se establece la necesidad de administrar un tratamiento complementario y se realiza el seguimiento durante largo tiempo, mucho más del que le dedican los primeros especialistas que la atendieron. No es infrecuente que las pacientes no vean más al ginecólogo o al cirujano por esta enfermedad, y el oncólogo constituye el único especialista con el que mantienen contacto para el seguimiento de la enfermedad. Las largas supervivencias actuales aconsejan que los médicos que en el futuro van a atender a estas mujeres por otros problemas de salud conozcan las características de la enfermedad y de los tratamientos que han seguido o siguen. Por ello, deberían conocer los principios básicos de la enfermedad para actuar de forma sinérgica y no antagónica en la atención integral de la paciente. Por tal razón, debe explicarse claramente en el ámbito docente el rol de médicos de familia, ginecólogos, cirujanos, oncólogos radioterapeutas y oncólogos médicos, sin olvidar el importante papel de los profesionales de las técnicas de imagen, la bioquímica, la farmacología, la epidemiología, la psicología o la biología molecular.

El ejemplo del cáncer de mama no es único. Se podría escribir lo mismo para el de pulmón, el de próstata o el del aparato digestivo, en los que numerosos médicos participan en su diagnóstico, tratamiento y seguimiento. ¿Cómo abordar esta situación desde el punto de vista docente? Parece obvio que los planes de estudio deberían imitar lo que ya sucede en la práctica clínica y considerar algunas enfermedades como unidades propias de enseñanza, más allá de los constreñimientos propios de las disciplinas académicas tradicionales. Ello no significaría la integración completa que algunos defienden, sino una aplicación más racional de los principios docentes que definirían espacios propios para las disciplinas, así como áreas comunes para los problemas de salud multidisciplinarios. La Oncología es, al respecto, un ejemplo de la necesidad práctica de este principio y una visión moderna de esta disciplina no puede contemplarla exclusivamente como una mera descripción de entidades patológicas, con su semiología, diagnóstico, pronóstico y tratamiento correspondiente. Es, en realidad, una materia que debe analizarse tanto de forma longitudinal como transversal, y esta consideración es de especial importancia para establecer su papel como disciplina docente. Analicemos estos conceptos con más detalle.

La Oncología debe considerarse como una disciplina con proyección longitudinal de pleno derecho. En este sentido existen una serie de contenidos propios, tanto conceptuales como prácticos, que tienen una entidad propia que debe enseñarse de forma específica. Por ejemplo, el proceso diagnóstico, la estadificación, la elección del protocolo terapéutico, el seguimiento de la enfermedad, la consideración a los factores emocionales o los aspectos biológicos de la enfermedad dotan a la Oncología de una identidad propia. A pesar de que inicialmente la enfermedad sea diagnosticada e incluso tratada por otros especialistas (ginecólogos y cirujanos en el cáncer de ovario, cirujano digestivo en el cáncer de colon), los pacientes que la sufren acaban en la consulta del oncólogo para seguir las pautas adecuadas de quimioterapia, hormonoterapia o radioterapia adyuvante, neoadyuvante o paliativa. Con frecuencia, los procesos diagnósticos y terapéuticos tienen unas características similares, lo que refuerza la necesidad de disponer de especialistas en el manejo de fármacos con índice terapéutico pequeño, pautas de tratamiento complejas y estrategias de seguimiento individualizadas. Todo ello acaba dando personalidad propia a una especialidad y la dota de un contenido nuclear que los estudiantes de medicina deben conocer.

Junto a estas actividades propias de la disciplina, la Oncología también comparte espacio docente y clínico con otras especialidades, tanto en el diagnóstico como en el tratamiento. La fre- 
Tabla. Organización transversal de la docencia: el ejemplo del cáncer de mama (entre paréntesis, las especialidades que podrían participar en la docencia)

Epidemiología: prevalencia, características poblaciones y factores de riesgo (Epidemiología y Salud Pública)

Concepto, presentación semiológica y exploración física (Ginecología)

Confirmación diagnóstica, clasificación y estadificación (Anatomía Patológica)

Técnicas de imagen en el diagnóstico y el seguimiento de la enfermedad (Radiología)

Principales marcadores tumorales y rol fisiopatológico (Bioquímica y Biología Molecular)

Proceso de decisión terapéutica (I): las posibilidades quirúrgicas (Ginecología/Cirugía)

Proceso de decisión terapéutica (II): la quimioterapia y la radioterapia (Oncología Médica/Oncología Radioterápica)

Proceso de decisión terapéutica (III): las ventajas e inconvenientes de los tratamientos antiestrogénicos y terapias dirigidas a diana (Oncología Médica)

Seguimiento de la enfermedad: el pronóstico, los controles periódicos y las señales de alarma (Cirugía, Ginecología Oncología Médica y Oncología Radioterápica)

cuente existencia de comités de tumores justifica la necesidad de considerar los pacientes con cáncer de forma global, de manera que pueda escogerse en cada caso la estrategia más oportuna. Por ejemplo, aunque la cirugía constituye el elemento terapéutico inicial para eliminar el elemento más visible del proceso, en ocasiones, las características del tumor aconsejan iniciar el tratamiento con quimioterapia primaria y continuar después con cirugía o radioterapia. Asimismo, algunas disciplinas básicas, como la bioquímica, la biología molecular, la genética, la anatomía patológica o la farmacología, muestran su auténtica importancia cuando se contemplan de forma integrada con la enfermedad neoplásica. La comprensión del ciclo celular, los procesos bioquímicos, la genética molecular, las alteraciones macro y microscópicas de los tejidos o la interferencia farmacológica de los procesos de división celular son mucho más fáciles de comprender en el contexto de la enfermedad neoplásica. Un plan de estudios racional debería contemplar de forma transversal la enfermedad neoplásica, sin olvidar asimismo elementos de comunicación (cómo dar malas noticias), psicológicos (consideración de factores emocionales) o epidemiológicos (prevención, factores de riesgo, pronóstico). Estos ejemplos demuestran cómo el interés de la Oncología médica se extiende por todo el plan de estudios desde la entrada de los estudiantes en la facultad hasta que salen de ella. A modo de ejemplo, la tabla resume cómo podría presentarse el cáncer de mama a los estudiantes.

\section{Conclusiones}

La docencia futura de la medicina obliga a un esfuerzo en la integración entre las disciplinas que se imparten en el ciclo básico y en el clínico. En la práctica esta posibilidad se encuentra dificultada por múltiples factores, entre los que destaca el interés de los profesores por no descuidar la impartición de lo que consideran la doctrina nuclear básica de su disciplina. El desarrollo de estrategias curriculares que permitan mantener este deseo con la necesidad de ofrecer una visión conjunta de los problemas de salud más prevalentes es muy deseable. En este sentido, la organización de los currículos para permitir la presentación integrada es posible, como muestra el ejemplo de la Oncología médica. Tales prin- 
cipios pueden ayudar de forma importante a la construcción de los nuevos planes de estudio de Medicina en los que la desaparición de las áreas de conocimiento, e incluso de la división en ciclos, puede constituir un estímulo para el debate estéril más que un elemento constructivo para replantear la docencia con la que formar a los futuros médicos. La consideración de espacios propios de las disciplinas, junto a la posibilidad de explicar integradamente los principales problemas de salud y enfermedades complejas de elevada prevalencia, debería ser un elemento importante en el debate.

El principio que defendemos es, pues, un modelo híbrido en el que coexistan la actividad docente dedicada a explicar las características de cada disciplina con asignaturas nuevas dedicadas a la presentación integrada de enfermedades cuyo diagnóstico y tratamiento escapan de los límites de una especialidad. Ello permitiría exponer de forma racional tales cuadros clínicos, lo que aproximaría la situación real a los estudiantes. La patología médica podría convertirse en el hilo conductor de esas actividades.

\section{Bibliografía}

1. López-Piñero JM. La medicina contemporánea: siglos XIX y xx. In López-Piñero JM. La medicina en la historia. Madrid: La Esfera de los Libros; 2002. p. 435-664.

2. Ackerknecht EH. A short history of medicine. Baltimore: Johns Hopkins University Press; 1982.

3. Tröhler U. Surgery (modern). In Bynum WF, Porter R, eds. Companion Encyclopedia of the history of medicine. Vol. 2. London: Routledge; 1993. p. 984-1028.

4. Baños JE, Bosch F. ¿Ha llegado, por fin, el lobo? La adaptación de los planes de estudio de Medicina al Espacio Europeo de Educación Superior: consideraciones en torno a una Orden Ministerial. Med Clin (Barc) 2008; 131: 457-9.

5. Nolla J, Coll J. L’adaptació a l'Espai Europeu d'Educació Superior: un camí amb tres reptes. Ann Med 2008; 91: 106-7.

6. Estrach MT. Ladaptació dels estudis de Medicina a l'Espai Europeu d'Educació Superior. Ann Med 2008; 91: 108-9.

7. Jové M, Castro A, Giralt M. Nou grau de Medicina a la Universitat Rovira i Virgili a partir d'un procés de reflexió entorn a l'Espai Europeu d'Educació Superior. Ann Med 2008; 91: 110-2.
8. Ribera J. Adaptació dels estudis de Medicina a l'Espai Europeu d'Educació Superior. Ann Med 2008; 91: 113-5.

9. Wood D. Problem-based learning. In Cantillon P, Hutchinson L, Wood D, eds. ABC of learning and teaching in medicine. London: BMJ Books; 2003. p. 8-11.

10. Velasco-Martín A, Alonso-Vielba J. Estado actual de la enseñanza de la Medicina. Valladolid: Secretariado de Publicaciones, Universidad; 1988.

11 Sarrias-Ramis R. Estudi de la implementació del pla d'estudis del 1993 de la Llicenciatura de Medicina de la Universitat Autònoma de Barcelona [tesis doctoral]. Bellaterra: Universitat Autònoma de Barcelona; 2000.

12. Alfonso MT, Fonseca M. Aprendizaje basado en problemas: situación actual y perspectivas de implantación en las facultades de medicina europeas. Dolor 2001; 16: 119-27.

13. Vivas NM, Badia A, Vila A, Baños JE. El aprendizaje basado en problemas como método docente en farmacología: la opinión de los estudiantes de medicina. Educ Med 2001; 4: 194-201.

14. Lucas M, García-Guasch R, Moret E, Llasera R, Melero A, Canet J. El aprendizaje basado en problemas aplicado a la asignatura de Anestesiología, Reanimación y Terapéutica del Dolor. Rev Esp Anest Anestesiol Reanim 2006; 53: 419-25.

15. Escanero JF, Guerra M, Ponce-Zumino A, Alda JO. Aprendizaje basado en la solución de problemas en fisiología circulatoria: el caso o problema como nexo de unión para la integración curricular. Educ Med 2002; 5: 191-4.

16. Osler W. Principles and practice of medicine. New York: Appleton; 1892.

17. Farreras-Valentí P. Medicina interna. 7 ed. Barcelona: Marín; 1967.

18. Rozman C. Prólogo a la novena edición. In Rozman C, ed. Medicina interna. Tomo I. Barcelona: Marín; 1978. p. XI-XIII.

19. Foz M. La dialéctica entre la medicina interna y las otras especialidades médicas desde la perspectiva profesional. Med Clin (Barc) 2007; 128: 141-7.

20. Borrador de la Orden Ministerial por la que se establecen los requisitos para la verificación de los títulos que habiliten para el ejercicio de la profesión de médico. 21 de noviembre de 2007.

21. Orden ECI/332/2008, de 13 de febrero, por la que se establecen los requisitos oficiales que habiliten para el ejercicio de la profesión de Médico. BOE n. ${ }^{\circ}$ 40, del 15 de febrero de 2008. p. 8351-5.

22. Terés J, Capdevila JA, Novell F, Cardellach F, Bundó M, Torres M. Formación troncal de las especialidades médicas: un reto del presente para una mejora del sistema sanitario. Med Clin (Barc) 2006; 127: 139-44.

23. Torres M, Cardellach F, Bundó M, Capdevila JA. Sistema 
formativo MIR: propuesta de cambios para la adecuación a las necesidades del modelo sanitario. Med Clin (Barc) 2008; 131: 777-82.

24. Jazieh AR, Henle K, Deloney LA, Savidge M, FulperSmith M, Nicholas R. The impact of cancer education program on the knowledge base of participating students. J Cancer Educ 2001; 16: 8-11.
25. Barton M, Bell P, Sabesan S, Koczwara B. What should doctors know about cancer? Undergraduate medical education from a societal perspective. Lancet Oncol 2006 7: 596-601.

26. Cruz-Hernández JJ. Docencia en Oncologia Médica. IX Congreso de la Sociedad Española de Oncología Médica. Libro de Resúmenes. Tenerife, 2000. p. 171-2. 